\title{
Colestasis y listeriosis en el tercer trimestre de la gestación
}

\section{Cholestasis and listeriosis in the third trimester of pregnancy}

\author{
I. Martínez-Montero, V. Segura Ortega, L. Martínez Jiménez, A. García Jiménez, \\ O. Unzetabarrenetxea Barrenetxea, A.F. Pérez Rodríguez
}

\section{RESUMEN}

La listeriosis es una infección producida por Listeria monocytogenes. Poco frecuente, afecta a personas en edades extremas de la vida, gestantes, inmunodeprimidos y, ocasionalmente, a individuos sanos

Su incidencia ha aumentado en los últimos años y presenta cierta tendencia a la estacionalidad, aumentando en verano. Puede aparecer de forma esporádica o a brotes.

En gestantes, la infección se produce más frecuentemente en el tercer trimestre y la clínica suele ser leve. Sin embargo, la infección sobre el feto es severa, pudiendo producir abortos, muertes fetales, corioamnionitis y partos pretérmino con recién nacidos infectados, manifestándose en forma de granulomatosis infantiséptica con abscesos y granulomas diseminados o, tardíamente, como meningitis y sepsis.

La colestasis intrahepática es una forma reversible de colestasis, de causa desconocida y específica de la gestación, siendo mas frecuente en multíparas, en el tercer trimestre y rara antes de la semana $26^{\mathrm{a}}$. Desaparece tras el parto y constituye la segunda causa de ictericia en la gestación, por detrás de la hepatitis.

El diagnóstico de colestasis es fundamentalmente clínico. Se manifiesta por prurito palmoplantar pero también puede cursar con náuseas, vómitos y molestias abdominales localizadas en hipocondrio derecho.

Dado que la listeriosis y colecistitis pueden compartir sintomatología, habrá que tener en cuenta la posibilidad de listeriosis para poner en marcha los mecanismos de confirmación diagnóstica (cultivo de fluidos o tejidos estériles: sangre, LCR neonatal, líquido amniótico o placenta) y el tratamiento específico precozmente.

Se presenta un caso de colestasis y listeriosis en el tercer trimestre con buen resultado maternofetal.

Palabras clave. Colestasis. Listeriosis. Gestación.

\section{ABSTRACT}

Listeriosis is an infection produced by Listeria monocytogenes. It is infrequent and affects people at extreme ages, pregnant women, immunocompromised people and occasionally, healthy people.

Its incidence has increased in recent years and shows a certain tendency to seasonality, increasing in summer. It can appear sporadically or as outbreaks.

In pregnant women the infection is most frequently produced in the third trimester and the symptoms are usually light. Nonetheless, the infection of the fetus is severe, and can produce miscarriages, fetal deaths, corioamnionitis and premature births with the newborn infected, manifested in the form of granulomatosis infantiseptica with abscesses and scattered granulomas or at a later stage, as meningitis or sepsis.

Intrahepatic cholestasis is a reversible form of cholestasis, its cause is unknown, it is specific to pregnancy and is more frequent in multiparous women, in the third trimester and rarely before the 26 th week. It disappears following childbirth and is the second cause of jaundice in pregnancy, after hepatitis.

The diagnosis of cholestasis is basically clinical. It appears as palmoplantar pruritus but can also produce nausea, vomiting and abdominal discomfort localized in the right hypochondrium.

Given that listeriosis and cholestasis can have a shared symptomology, the possibility of listeriosis must be borne in mind in order for early implementation of the mechanisms of diagnostic confirmation (cultivation of sterile fluids or tissues: blood, neonatal CSF, amniotic liquid or placenta) and specific treatment.

We present a case of cholestasis and listeriosis in the third trimester with a good maternofetal result.

Key words. Cholestasis. Listeriosis. Pregnancy.
Servicio de Obstetricia y Ginecología. Complejo Hospitalario de Navarra. Pamplona.

Recepción: 25 de julio de 2013

Aceptación provisional: 3 de septiembre de 2013

Aceptación definitiva: 30 de septiembre de 2013

\section{Correspondencia:}

Isabel Martínez Montero

Servicio de Obstetricia y Ginecología

Complejo Hospitalario de Navarra

C/ Irunlarrea, 4

31008 Pamplona

isabel.martinez.montero@cfnavarra.es 


\section{INTRODUCCIÓN}

Listeria monocytogenes es un bacilo gram positivo, móvil, anaerobio facultativo. Se encuentra ampliamente distribuido en la naturaleza y es capaz de sobrevivir bajo condiciones extremas de $\mathrm{pH}$, salinidad y temperatura, existiendo gran dificultad en su prevención ${ }^{1-3}$. Se trata de un microorganismo intracelular oportunista ${ }^{4,5}$.

Se transmite principalmente por ingesta de alimentos contaminados, siendo los más frecuentes los productos cárnicos precocinados, leche y productos lácteos no pasteurizados, verduras mal lavadas, embutidos y marisco ${ }^{1,5-8}$. Esta bacteria enteropatógena tiene especial tropismo por la placenta y el SNC ${ }^{5,6,9-11}$, y es destruida con la pasteurización y por la mayoría de los agentes desinfectantes ${ }^{3,9}$.

La incidencia de listeriosis en el embarazo es variable, estimándose que afecta a 0,2-0,8/1.000 partos ${ }^{2,11}$; pero, en cualquier caso, se trata de una infección infradiagnosticada.

Las manifestaciones clínicas de la listeriosis pueden diferir sustancialmente según el estado inmunitario del individuo infectado. La clínica de la listeriosis en la gestante, aunque inmunocompetente, suele ser leve y cursa habitualmente con fiebre y cuadro pseudogripal inespecífico y, hasta en un $20 \%$ de los casos, pueden aparecer síntomas gastrointestinales (diarrea, dolor abdominal, etc.) que, generalmente, preceden al episodio febril ${ }^{2,3}$. El compromiso del SNC es infrecuente y, por el contrario, pueden cursar sin síntomas hasta el $29 \%$ de los $\operatorname{casos}^{7,9}$. Sin embargo, puede tener graves repercusiones fetales.

La infección congénita por Listeria presenta globalmente un $40-50 \%$ de mortalidad fetal o neonatal. Si la infección tiene lugar en el primer o segundo trimestre, lo que ocurre en el $20 \%$ de los casos, provoca abortos sépticos y muerte fetal intrauteri$\mathrm{na}^{10,11}$. En el tercer trimestre $(80 \%$ de los casos), produce en dos tercios de los mismos corioamnionitis y parto prematuro con un $20 \%$ de mortalidad perinatal. En el tercio restante puede cursar de forma asintomática en el feto/ neonato ${ }^{2,7}$.
Es frecuente el líquido amniótico pigmentado, pardusco o teñido de meconio en caso de infección fetal, aún en los embarazos pretérmino, constituyendo un hallazgo típico de listeriosis y que hace pensar en su diagnóstico ${ }^{12,13}$.

La infección neonatal se puede manifestar en forma de listeriosis neonatal precoz o tardía $a^{2-4}$. La infección materna puede ser difícil de diagnosticar debido a las manifestaciones sistémicas inespecíficas. La confirmación diagnóstica se realiza mediante cultivo de fluidos o tejidos estériles (sangre, LCR neonatal, líquido amniótico o placenta) ${ }^{1,7,14}$. Ante una gestante con fiebre superior a $38^{\circ} \mathrm{C}$ se debe realizar una analítica sanguínea que incluya hemograma y PCR. Un incremento de la PCR acompañado de leucocitosis en ausencia de otra focalidad, debe orientar hacia el diagnóstico de listeriosis, estando indicada la realización de un hemocultivo coincidiendo con el pico febril ${ }^{1,2,7,15}$.

El tratamiento antimicrobiano tiene por objetivo mejorar la evolución fetal/ neonatal. Una terapia apropiada y precoz con ampicilina y gentamicina durante una semana mejoran la evolución y pueden revertir el cuadro $^{1,2,11,14,16-18}$.

La colestasis intrahepática es una forma reversible de colestasis específica de la gestación, que aparece en el tercer trimestre siendo muy rara antes de la semana $26^{\mathrm{a}}$, y que desaparece tras el parto ${ }^{1,9,19-22}$. Tiene una prevalencia de $0,7 \%$ de embarazos y constituye la segunda causa de ictericia en la gestación, por detrás de la hepatitis y por delante de la litiasis biliar.

Es más frecuente en multíparas, muy rara en las razas oriental y negra y, aunque tiene una amplia variabilidad geográfica, su máxima incidencia se produce en Chile $^{6,23}$.

Su etiología es desconocida y su diagnóstico, fundamentalmente, clínico. Su principal síntoma es el prurito palmoplantar y posteriormente generalizado, con predominio nocturno provocando insomnio e irritabilidad; sin embargo, también puede ocasionar náuseas, vómitos y molestias abdominales localizadas en hipocondrio derecho. 
La elevación de los ácidos biliares es la prueba más sensible. También pueden aumentar las tasas de bilirrubina total, fosfatasas alcalinas, transaminasas, GGT, colesterol, y el índice de protrombina ser menor de $70 \%$. Cuando las pruebas de laboratorio son normales pero la clínica es sugestiva de colestasis, se recomienda repetir la analítica en un plazo de 2 semanas.

El diagnóstico diferencial deberá hacerse fundamentalmente con hepatitis virales y tóxicas, hepatitis autoinmunes, cirrosis, colangitis, coledocolitiasis, esteatosis, esteatohepatitis, dermatopatías propias de la gestación y reacciones alérgicas. Igualmente habrá que hacer diagnóstico diferencial con preeclampsia (síndrome de Hellp) e hígado graso ${ }^{20}$.

Debido a que la vesícula biliar, el hígado y el intestino son reservorios de Listeria, la listeriosis puede compartir sintomatología con la colecistitis, que es, por otra parte, una de las causas más frecuentes de dolor abdominal en la mujer embarazada. Se caracteriza por dolor de aparición brusca en hipocondrio derecho acompañado de fiebre y leucocitosis, pudiéndose acompañar también de náuseas, vómitos, fiebre, taquicardia y taquipnea lo que puede llevar a pensar que se trata de un cuadro de origen respiratorio como puede ser una neumonía basal. Su frecuencia es variable, oscilando entre $1 / 1.500$ y $1 / 10.000$ embarazos $^{21}$.

La gestación produce cambios en la producción de bilis e induce la formación de cálculos biliares ${ }^{21,23}$. El procedimiento diagnóstico más útil para la colelitiasis/ colecistitis es la ecografía hepatobiliar que llega a alcanzar una sensibilidad del $88 \%$ y especificidad del $80 \%^{20,21,24}$.

\section{CASO CLÍNICO}

Secundigesta de 40 años de edad, natural de Colombia, con antecedente de embarazo y parto anterior sin complicaciones que presenta obesidad mórbida (IMC 43) y cólicos biliares de repetición. En la semana 30+ 4 de gestación acudió al servicio de Urgencias por presentar prurito generalizado de 48 horas de evolución y cuadro pseudogripal con febrícula, artromialgias y malestar general. En la exploración clínica se objetivaron lesiones de rascado sin lesiones urticariformes y febrícula de $37^{\circ} \mathrm{C}$. Se constató el bienestar fetal mediante ecografía obstétrica (feto en cefálica dorso anterior, placenta posterior normoinserta, y líquido amniótico normal) y registro cardiotocográfico (patrón reactivo, adinamia). Se solicitó hemograma que no evidenció alteraciones significativas, excepto anemia moderada ( $\mathrm{Hb} 10 \mathrm{~g} / \mathrm{dl}$ ), elevación de transaminasas (AST y ALT 208 y 238 U/L) y ácidos biliares $56,9 \mu \mathrm{mol} / \mathrm{L}$. El estudio de coagulación fue normal. Se decidió el ingreso de la paciente por colestasis gravídica y se inició tratamiento con ácido ursodesoxicólico a dosis de $13 \mathrm{mg} /$ $\mathrm{kg} / \mathrm{día}$, loratadina $10 \mathrm{mg} / 24 \mathrm{~h}$ y colestiramina 3 $\mathrm{gr} / 8 \mathrm{~h}$. A las 48 horas de su ingreso la paciente comenzó con fiebre de hasta $38,6{ }^{\circ} \mathrm{C}$, tos no productiva, disnea y otalgia, siendo la auscultación broncopulmonar normal y la saturación de $98 \%$. Se solicitó radiografía de tórax, hemocultivos y frotis nasofaríngeo (PCR gripe) y se inició tratamiento empírico con ampicilina $2 \mathrm{~g} / 4 \mathrm{~h}$, gentamicina $240 \mathrm{mg} / 24 \mathrm{~h}$ y oseltamivir $75 \mathrm{mg}$. Al mismo tiempo, se solicitó ecografía abdominal, que fue normal, para descartar colelitiasis/colecistitis dada la frecuente asociación de ambos procesos con colestasis, sobre todo en mujeres obesas y multíparas, condiciones que cumplía la paciente. Ante la evolución analítica y los antecedentes de cólicos biliares, se solicitó una segunda ecografía que, en esta ocasión, confirmó el diagnóstico de colelitiasis.

El frotis nasofaríngeo para gripe resultó negativo y en el hemocultivo se aisló Listeria monocytogenes sensible a ampicilina, gentamicina y penicilina por lo que se suspendió oseltamivir y se continuó con el tratamiento antibiótico pautado. Así mismo, se suspendió el tratamiento con preparados orales de hierro.

La paciente presentó una evolución tórpida del cuadro de colestasis con descenso parcial de las transaminasas y episodios aislados de prurito a pesar del tratamiento.

Las determinaciones analíticas sucesivas evidenciaron modificaciones con elevaciones y descensos de transaminasas y ácidos biliares, tal y como se recoge en la tabla 1 .

El cuadro de listeriosis presentó una evolución favorable, permaneciendo apirética y evidenciándose el descenso paulatino de PCR desde el inicio del tratamiento antibiótico (Tabla 1).

Se realizó control de bienestar fetal seriado mediante ecografía obstétrica y registro cardiotocográfico hasta la semana $32+4$, en la que se inició de forma espontánea el trabajo de parto tras completar 14 días de tratamiento con ampicilina y 7 días con gentamicina, ambas por vía endovenosa. 
Tabla 1. Evolución analítica de la paciente

\begin{tabular}{ccccccc}
\hline $\begin{array}{c}\text { Semanas } \\
\text { Gestación }\end{array}$ & Leucocitos/ $\mathbf{\mu L}$ & $\begin{array}{c}\text { Neutrófilos } \\
(\%)\end{array}$ & $\begin{array}{c}\text { PCR } \\
(\mathbf{m g} / \mathbf{d L})\end{array}$ & AST (U/L) & ALT (U/L) & $\begin{array}{c}\text { Ácidos Biliares } \\
(\boldsymbol{\mu m o l} / \mathbf{L})\end{array}$ \\
\hline $30+4$ & 5.700 & 75,1 & 2,76 & 208 & 238 & 56,9 \\
\hline $30+5$ & 6.600 & 72,8 & 5,39 & 160 & 187 & 13,5 \\
\hline $30+6$ & 6.600 & 77,6 & 7,18 & 122 & 170 & 28,5 \\
\hline $31+1$ & 6.000 & 70,9 & 4,53 & 60 & 90 & 11,8 \\
\hline $31+5$ & 6.800 & 62,7 & - & 217 & 176 & 58,8 \\
\hline $32+3$ & 7.600 & 64,5 & 0,5 & 348 & 359 & 0,7 \\
\hline Puerperio & 6.300 & 49,5 & - & 22 & 14 & \\
\hline
\end{tabular}

Se comprobó bienestar fetal intraparto mediante monitorización maternofetal, objetivando líquido amniótico meconial. El parto fue eutócico, obteniendo una niña de $2.225 \mathrm{~g}$ de peso, test de Apgar 9/10 y pH de cordón 7,36 arterial y 7,37 venoso.

La RN ingresó en neonatología por prematuridad y antecedente de listeriosis materna. Los cultivos que se le realizaron (frotis faríngeo, vermis, umbilical, hemocultivo, urocultivo y coprocultivo) fueron negativos, siendo dada de alta a los 10 días de vida. La evolución puerperal fue normal con alta a las 48 horas del parto.

El examen anatomopatológico de la placenta evidenció múltiples infartos "sépticos" con presencia de aisladas formaciones bacilares Gram positivas, compatible con estado de infección por Listeria.

\section{DISCUSIÓN}

La listeriosis es una enfermedad infrecuente de declaración obligatoria. La contaminación alimentaria afectando a una población en estado de inmunodepresión, tales como RN, ancianos, pacientes con hepatopatía, gestación, etc. constituyen los dos factores fundamentales para la presentación de la enfermedad ${ }^{1,5,12,17}$, tanto en forma epidémica como en casos aislados.

El periodo de incubación es variable, oscilando entre 2 y 6 semanas y el mecanismo de transmisión sería la contaminación de alimentos (leche pasteurizada, verduras, mariscos, quesos, carnes crudas y alimentos congelados prefabricados) con materias fecales procedentes de los reser$\operatorname{vorios}^{1-3,7,9,15,16}$. Listeria en un huésped con inmunidad normal puede desarrollar una infección banal e incluso asintomática y hacer que permanezca como reservorio o portador asintomático en la mucosa faríngea y tracto digestivo ${ }^{2}$.

Desde el punto de vista clínico se caracteriza por presentar un variado espectro de formas de presentación. Los síntomas como fiebre, artromialgias, cefaleas, otalgia, tos, náuseas, vómitos y el hallazgo analítico de leucocitos pueden inducir al diagnóstico de cuadros pseudogripales.

Sin embargo, siempre hay que sospechar la existencia de listeriosis ante historia de prematuridad, feto muerto y cuadros febriles con sintomatología inespecífi$\mathrm{ca}^{2,7,9,11}$

En el caso que aquí se presenta, el motivo del ingreso no fue la sintomatología pseudogripal de la gestante sino el prurito generalizado que hizo sospechar que se tratase de una colestasis de embarazo con el consiguiente riesgo fetal que conlle$\mathrm{va}^{20}$. Dado que en el momento de acudir al servicio de Urgencias estaba apirética, tal vez no hubiera ingresado hasta que hubiese presentado el pico febril que presentó posteriormente y se hubiese demorado el diagnóstico y el tratamiento.

Si bien la vesícula biliar, el hígado y el intestino son reservorios de Listeria $^{9,21,23}$, en el caso presentado no se puede descartar que no sean dos cuadros coincidentes y no consecuencia la listeriosis de la colelitiasis, ya que la paciente había presentado cólicos biliares por colelitiasis con anterioridad al embarazo; colelitiasis que pos- 
teriormente fue confirmada en la segunda ecografía realizada.

A pesar del diagnóstico temprano y el tratamiento antibiótico adecuado en tiempo y dosis óptimos, así como la suspensión del hierro oral que tomaba la paciente, se produjo un parto prematuro y líquido amniótico meconial sin signos de sufrimiento fetal, ambas complicaciones típicas de la listeriosis en el tercer trimestre ${ }^{2,11,12}$.

La incidencia de la listeriosis en la gestación es 17 veces más alta que en la población general, lo que se atribuye a que Listeria es un microorganismo intracelular, y que en las mujeres embarazadas se produce una disminución de la inmunidad mediada por células $\mathrm{T}^{4,5}$.

La colestasis ocurre más frecuentemente en el tercer trimestre de la gestación ${ }^{5,17,18}$, tal y como ocurrió en el caso presentado.

En este caso, se llegó al diagnóstico de certeza, como habitualmente ocurre ${ }^{3,7}$, mediante los hemocultivos realizados durante el pico febril que presentó la gestante durante su ingreso. Tras la extracción de la muestra para el hemocultivo se inició tratamiento antibiótico endovenoso empírico y sólo la confirmación diagnóstica de listeriosis mediante el hemocultivo permitió llevar a cabo su tratamiento específico.

En el caso que se presenta, fue necesario repetir la ecografía para demostrar la existencia de colelitiasis.

Resulta fundamental llegar precozmente al diagnóstico de certeza, ya que el tratamiento adecuado reduce la morbimortalidad materno-fetal y mejora el pronóstico fetal. A diferencia de otras causas de corioamnionitis, en las cuales la inducción del parto es el abordaje estándar, la listeriosis puede ser tratada con antibioticoterapia a altas dosis para resolver la infección, obviando la finalización del embarazo.

La asociación de ampicilina y gentamicina ejerce un efecto sinérgico y cubre otros posibles gérmenes. Se recomienda su uso hasta la mejoría de los síntomas, generalmente durante una semana ${ }^{2,3,4,11,14-18}$. En casos de alergia a penicilina, la eritromicina, igualmente asociada a gentamicina, es el fármaco de segunda elección. En el caso presentado, el resultado final fue un recién nacido prematuro sano con una buena evolución posterior sin signos de infección, a pesar de que el estudio anatomopatológico de la placenta evidenció la presencia de microabscesos típicos de la listerio$\operatorname{sis}^{1,2,7,11,13,23,25,26}$.

Por otra parte, cabe destacar la necesidad de prevención primaria en cuanto a la infección por Listeria. Puesto que no se dispone de vacuna frente a la listeriosis ${ }^{26}$ se debe instruir a las embarazadas con una serie de recomendaciones (Tabla 2) ${ }^{27,30}$.

Tabla 2. Recomendaciones del CDC para la prevención de la listeriosis

\section{Recomendaciones generales:}

- Cocinar los alimentos crudos de origen animal.

- Lavar minuciosamente los vegetales crudos antes de consumirlos.

- Separar las carnes crudas de los vegetales y de los alimentos cocidos y los que vienen listos para consumir.

- Evitar consumir leche o queso sin pasteurizar y otros alimentos hechos con este tipo de leche.

- Lavarse las manos, cuchillos y tablas de cortar después de manipular alimentos crudos.

- Consumir los alimentos perecederos y los listos para consumir antes de la fecha de expiración.

Más recomendaciones para las personas con alto riesgo, como mujeres embarazadas:

- No comer quesos blandos, quesos azules o quesos estilo mexicano, a menos que en la etiqueta se indique que están hechos con leche pasteurizada.

- No comer perritos calientes, carnes frías ni fiambres, a menos que los haya recalentado a altas temperaturas. Lavarse las manos después de manipular estos alimentos.

- No comer patés ni untables de carne refrigerados (sí los enlatados o envasados).

- No consumir mariscos ahumados refrigerados a menos que se hayan cocido. Se pueden consumir los mariscos ahumados enlatados o envasados.

Fuente: Centers for Disease Control and Prevention (CDC). Web site, Listeriosis technical information and FAQs www.cdc.gov/ncidod/dbmd/diseaseinfo/ listeriosis_t.htm 


\section{BIBLIOGRAFÍA}

1. Deguelte S, Metge M-P, Quereux C, Gabriel R. Listerioisis au tours de la grosseasse. Encyclopedie Médico-Chirúrgicale S-039-E-10 (2004).

2. Giвв D. Listeria Monocytogenes. En: Anne Greenough, Johb Osborne, Sheena Sutherland. Infecciones congénitas, perinatales y neonatales. Barcelona Ed Masson SA, 1995: 135-143.

3. Suy Franch A, Jaramago J. Infecciones bacterianas. En: Fabre Gonzalez E. Asistencia a las complicaciones médicas y quirúrgicas del embarazo. Madrid: Adalia, 2010: Tomo II, 1-23.

4. Torres K, Sierra S, Poutou R, Carrascal A, MerCADO M. Patogénesis de Listeria monocytogenes, microorganismo zoonótico emergente. Rev MVZ Cordoba 2005; 10: 511-543.

5. Eguiluz Gutiérrez-Barquin I, Barber Marrero Mi, Cabrera Morales F, Valle Morales L, García HERNANDEZ JA. Infección perinatal por Listeria monocytogenes durante el embarazo. Prog Obstet Ginecol 2007; 50: 457-466.

6. Cisternas A, Lagos N, Galstuch J, González C, GARCía C, DíAz J. Infección por Listeria monocytogenes y embarazo con buen resultado perinatal. Caso clínico. Rev Chil Obstet Ginecol 2002: 67: 237-241.

7. Ovalle Solís A. Infecciones bacterianas y protozoarias durante el embarazo. En: Gratacós E y col. Medicina Fetal. Buenos Aires. Madrid 8 2009) Ed. Panamericana 2007: 549-554.

8. Doblas PA, Egulluz I, Barber MA, Hijano JV, Gómez-Castellano M, Monis S et al. Listeriosis y gestación. Clin Invest Gin Obstr 2002; 29: 233-237.

9. Larrain de la Cruz D, Abarzuza F, de Jourdan F, Merino P, Belmar C, García P. Infecciones por Listeria monocytogenes en mujeres embarazadas: experiencia del Hospital Clínico de la Pontificia. Universidad Católica de Chile. Rev Chil Infectol 2008; 25: 336-341.

10. Parkassh V, Morroti RA, Joshi V, Cartun R, RAUCH CA, West AB. Inmunohistochemical detection of Listeria. Antigen in the Placenta in perinatal Listeriosis. Int J Gun Pathol 1998; 17: 343-350.

11. Mattew Miller J, Pastortek J. Listeriosis. En: Buttino, Elkayam, Evans, Galbraith, Gall, Sibai. Tratamiento de las complicaciones clínicas del embarazo, $3^{\mathrm{a}}$ edición. Buenos Aires (Argentina). Ed. Panamericana 2000: 783-785.
12. Cruz M, Carretero P, Cámara M, Fernández J, CAÑo A, GiménEz F. Listeriosis durante la gestación. Un problema diagnóstico. Prog Obstet Ginecol 2002: 45: 265-268.

13. Кoн KS, Cole TL, Orkin AJ. Listeria Amnionitis as a case of fetal distress. Am J Obstet Gynecol 1980; 136: 261-263.

14. Palacián Ruiz MP, Cameo Rico Mi, Arazo Garcés P, Marco Lamata ML, Revillo Pinilla MJ. Infecciones por Listeria monocytogenes. Rev Esp Quimioter 2011; 24: 112-114.

15. Goncé A, García L, López M. Listeria y gestación. Protocols Medicina Fetal y Perinatal. Servei de Medicina MaternoFetal. ICGONHospital Clinic Barcelona, junio 2012.

16. Lamont RF, Sobel J, Mazaki-Tovi S, Kussanovic JP, Vaisbuch E, KIm SK et al. Listeriosis in human pregnancy: a systematic review. J Perinat Med 2011; 39: 227-236.

17. Arias M, Nuno M, Noval M, Fonseca A, Menéndez C. Listeriosis en el adulto. Revisión de 10 casos. An Med Interna 2004; 21: 75-78.

18. Illescas T, Viana R, Cabrillo E, González A. Bacteriemia por Listeria en el tercer trimestre del embarazo. Prog Obstet Ginecol 2005; 48: 602-605.

19. García M, García A, Pérez C, Grande J, HernánDEZ JM, DE LA FuENTE P. Colestasis intrahepática del embarazo: diagnóstico y manejo. Prog Obst Ginecol 2005; 48: 189-191.

20. Hepatopatías y embarazo. Protocolo de la Sociedad de Ginecología y Obstetricia, actualizado en 2006.

21. Lailla Vicens JM, Marimón Garcia E, Boguña Ponsa JM. Enfermedades de las vías biliares y páncreas. En: Fabre Gonzalez E. Asistencia a las complicaciones médicas y quirúrgicas del embarazo. Madrid: Adalia, 2010: Tomo I, 363-372.

22. Pérez Rodríguez AF, Roche Roche M, Larrañaga AzcÁrate C. Patología médica y embarazo. Trastornos gastrointestinales, neurológicos, cardiovasculares y dermatológicos. An Sist Sanit Navar 2009; 32 (Supl. 1): 135-157.

23. Valdivieso V, Covarrubias C, Seigel F. Pregnancy and cholelithiasis: Pathogenesis and natural course of gallstones diagnosed in early puerperium. Hepatology 1993; 17: 1-7.

24. del Cura Rodríguez JL, Pedraza Gutiérrez S, GAyete CARA A. Radiología esencial. SERAM Sociedad Española de Radiología Médica. Vol 1. Buenos Aires. Ed. Panamericana 2010: 530.

25. Topalovski M, Yang SS; BoonPast Y. Listeriosis of the placenta. Clinicopathologic study of seven cases. Am J Obstec Ginecol 1993; 169: 616-620. 
26. Cunninghan F, Leveno K, Bloom S, Hauthu J, RouSE D, SPOng C. Enfermedades infecciosas. En Williams Obstetrics. Complicaciones médicas y quirúrgicas. $23^{\text {a }}$ edición. MC Graawhill Medical. Interamericana. NewYork 2010: 1224-1225.

27. Centers for Disease Control and Prevention (CDC). Web site, Listeriosis technical information and FAQs www.cdc.gov/ncidod/ dbmd/diseaseinfo/listeriosis_t.htm

28. Tappero JW, Schuchat A, Deaver KA, Mascola L, WENGER JD. Reduction in the incidence of hu- man listeriosis in the United States. Effectiveness of prevention efforts? The Listeriosis Study Group. Jama 1995; 273: 1118-1122.

29. Ogunmodede F, Jones JL, Scheftel J, Kirkland E, SchULKIN J, LyNFIELD R. Listeriosis prevention knowledge among pregnant women in the USA. Infect Dis Obstet Gynecol 2005; 13: 1115.

30. Gelfand M. Treatment, prognosis, and prevention of Listeria monocytogenes infection. En UpToDate, 2013. Ed Sexton DJ, Edwards MS. www.uptodate.com@2013UpToDate. 
